\title{
Application of the cyclostationairity for the cutting tool diagnosis
}

\author{
Khalid Ait-Sghir $^{1, a}$, Mohamed El Badaoui ${ }^{2}$, François Guillet $^{2}$, Driss Aboutajdine $^{3}$ \\ AND JEAN-PAUl Dron ${ }^{1}$ \\ ${ }^{1}$ Groupe de Recherche en Science Pour l'Ingénieur, Université de Reims Champagne Ardenne, UFR Sciences Exactes \\ et Naturelles, Moulin de la Housse, BP 1039, 51687 Reims Cedex 2, France \\ 2 Université de Lyon, Université Jean Monnet de Saint Etienne, Campus Roannais, Laboratoire d'Analyse des Signaux \\ et des Processus Industriels (LASPI), 42300 Roanne, France \\ 3 Faculté des sciences, Université Mohammed V-Agdal, 4 avenue Ibn Battouta, Rabat, Maroc
}

Received 13 January 2014, Accepted 15 May 2014

\begin{abstract}
This work is interested to the analysis of the vibratory signals coming from a milling operation. The objective is the detection of cutting tool breakage using the cyclostationary tools. Initially, we will show that the vibration signals captured from the milling operation are cyclostationary. The proposed cyclostationary methods are the first and second order synchronous statistics and the spectral correlation. A test rig, composed of a milling machine (cutter with 5 teeth) and a workpiece, is used to extract the vibration signals that are angular sampled in the free fault case and one broken tooth case. This test rig is instrumented with three accelerometers, installed in the three directions, and an optical encoder that allows the angular sampling. Then we will see that the angular sampling of the signals captured from a milling operation is essential to preserve the cyclostationary properties destroyed, in the case of the temporal sampling, by speed fluctuations. The proposed method capacity to detect the broken tooth is shown. The synchronous statistics of order 1 and order 2 detect the broken tooth presence and its emplacement. The spectral correlation analysis distinguishes the broken tooth presence, but is not practical for the diagnosis. For that, an indicator based on the spectral correlation is proposed.
\end{abstract}

Key words: Vibration analysis / cyclostationarity / cutting tools / spectral correlation / synchronous statistics

\section{Introduction}

The production systems optimization is an ongoing concern in the industry, mainly in sector of machining where the economic stakes are high. Today, machining centers have become relatively autonomous and accordingly execute the production in an automatic way. However, progress is still to be performing in control of the final workpiece quality and the detection of broken tools.

The tool breakage is the major cause of unexpected stoppage in machining, which means not only in terms of time lost, but also in terms of the degradations generated, sometimes important [1]. In an average machine tool, the amount of downtime caused by tool breakage is estimated at $6.8 \%$ [2] while others estimate up to $20 \%$ [3]. Also add that even if there is no tool breakage, use of damaged or worn tools can cause a loss in the final workpiece quality. Hence there is a need to establish a system for monitoring

\footnotetext{
a Corresponding author:

khalid.ait-sghir@univ-reims.fr
}

and diagnosis of the cutting tools. The use of an accurate and reliable tool condition monitoring system has several virtues [4]: an increase in cutting speed from 10 to $50 \%$, a reduction of unscheduled downtime and a decrease in the overall cost 10 to $40 \%$. The service life of the tool can also be increased by 10 to $65 \%$ [5]. A potential improvement can be obtained by detecting poor machining conditions.

In general, a tool condition monitoring system (TCMS) integrates three essential parts (Fig. 1):

- The first includes the choice of physical quantities to observe (one or more sensors) and data acquisition.

- The second concerns the extraction of the information from the acquired signals. By using the cyclostationarity tools to provide relevant indicators, this part will be the purpose of the work.

- Based on the extracted information, the final part concerns the decision strategy on the machine status.

The success of a TCMS is performed through the development of signal processing techniques that can get 


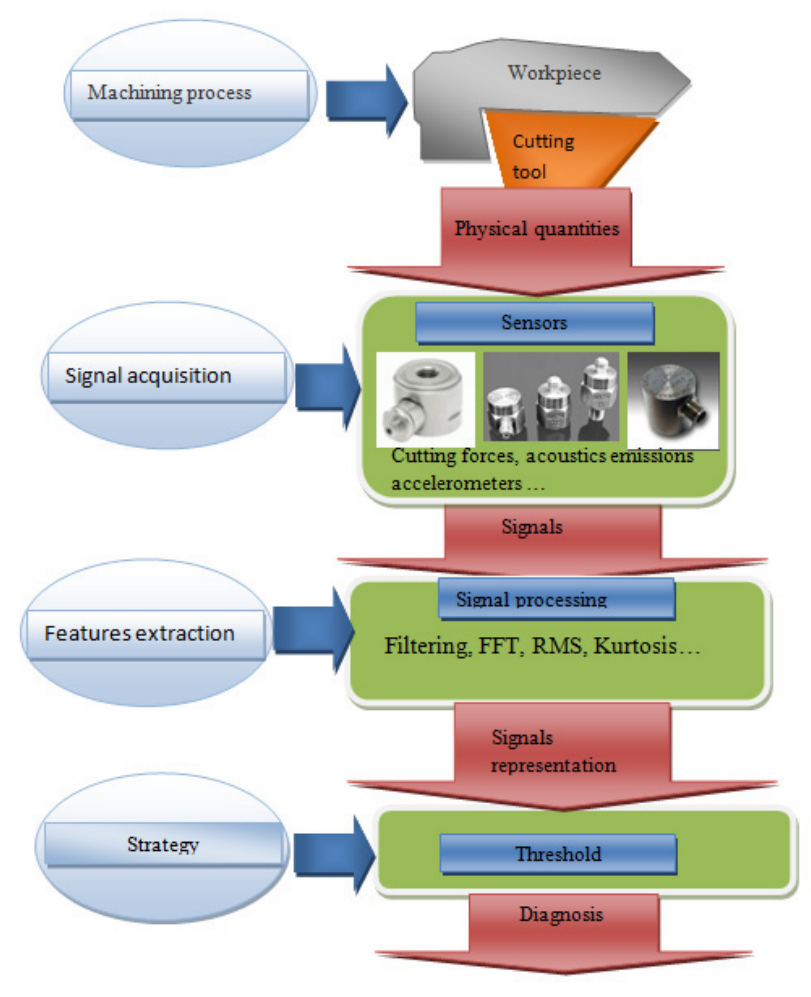

Fig. 1. Schematic representation of tool condition monitoring system.

maximum information, of the cutting tools condition, from the acquired signals.

Several parameters have been proposed for the cutting tool condition monitoring. We find the cutting force $[6,7]$, the vibratory signature [8-10], temperature [11] and acoustic emission [12]. Among these approaches, the vibration analysis has taken, recently, considerable importance because of the rich information that it contains, and development of techniques more sophisticated of instrumentation and signal processing. In this work, we focus our study on the vibration analysis of a milling machine. This type of machine operates cyclically with one or several basic cycles, which repeat indefinitely and relating to periodic characteristics at different generated signals. This type of signal is a class called cyclostationary process which is characterized by the periodicity of their statistical parameters (the statistical moments are periodic at different order). This characteristic is particularly obvious in vibrations generated by the milling process since the attack on the workpiece metal by each of the L cutter teeth, is repeated $\mathrm{L}$ times per revolution. Thus these generated vibratory signals possess the cyclostationarity character that we take care to synchronize with the spindle rotation (angular analysis).

The cyclostationarity notion has been developed, since 40 years, in the telecommunication domain by researchers, especially by Gardner $[13,14]$. Their results have been exploited recently in vibration analysis in order to diagnosis the rotating machines (gear, bearings, diesel motor) [15-17]. For these machines, the cyclostationarity exploitation still remains rich and attractive. However, for the machining cutting tools, such a milling, this intrinsic property has not been used before in research's work. Either the research's works use classical tools as spectral analysis which assumes the stationarity of the vibration signals, or by exploiting the non-stationary tools such as the wavelet analysis which considers the signals as nonstationary. For these reasons, we will exploit the cyclostationarity property and consider it for the diagnosis of cutting tools.

The paper is organized as follows. In Section 2, the cyclostationary tools are introduced and their essentials are explained. In Section 3, a model of milling signals is presented. In Section 4 the experimental setup, is exposed. In Section 5 the signals analysis is shown and the conclusion is given at the end.

\section{Cyclostationarity and cyclostationary tools}

\subsection{Cyclostationarity}

The term "cyclostationnary" refers to a subclass of nonstationary process whose statistics periodically vary with a generic variable (time, angle). These processes are random in nature but exhibit periodicity in their statistics.

A signal $x(t)$ is a first order cyclostationary (CS1) with respect to the period $T$, if its moment at first order (mean) is periodic

$$
m_{x}(t)=E\{x(t)\}=m_{x}(t+T)
$$

with $E\{\cdot\}$ denotes the statistical expectation operator.

A signal $x(t)$ is a second order cyclostationary (CS2) with respect to the period $T$, if its moment at second order is periodic

$$
R_{2 x}\left(t_{1}, t_{2}\right)=E\left\{x^{*}\left(t_{1}\right) x\left(t_{2}\right)\right\}=R_{2 x}\left(t_{1}+T, t_{2}+T\right)
$$

The signals that are both CS1 and CS2 are called wide sense cyclostationary.

A signal $x(t)$ is a $k$ order cyclostationary (CSk) with respect to the period $T$, if its moment at $k$ order is periodic.

We have defined the stochastic approach of the cyclostationarity. However, to be applied to the real signals it is necessary to assume that the signals are cycloergodic [17]. If the signal is assumed cycloergodic, the statistical expectation operator (Eq. (1)) can be replaced by the time average operator which can be defined for a discrete process as:

$$
\langle x(n)\rangle=\lim _{M \rightarrow \infty} \frac{1}{(2 M)+1} \sum_{i=-M}^{M} x(i)
$$




\subsection{Synchronous average}

Let $x(t)$ be a cyclostationary signal with a cyclic pe$\operatorname{riod} T$, which has a number of cycles $N$. The synchronous average is given by the following equation:

$$
m_{T}^{N}(t)=\frac{1}{N} \sum_{k=0}^{N-1} x[\bmod (t+k T, N T)]
$$

where $\bmod (a, b)$ is the remainder of division of $a$ by $b$. The function mod is used to define the synchronous average for all $t$. For its estimation, the signal is divided in sections equal to basis cycle machine. Then these sections are averaged. Although the synchronous average is a simple tool, a number of points must be considered. The first point relates to the period that be accurately known, else the synchronous average tend to 0 . The number of points per period must be an integer and the last point is that the signals have to be truncated in order to have a finite number of periods. In this paper, these conditions are satisfied by the fact that the signals are angular sampled and have a constant number of points per spindle revolution. Thus the signals are synchronized with respect to the cycle period.

\subsection{Synchronous variance}

Synchronous variance is a second order cyclostationary tool. It is defined by the following equation:

$$
v_{T}^{N}(t)=\frac{1}{N} \sum_{k=0}^{N-1}\left(x\left(\bmod (t+k T, N T)-m_{T}^{N}(t)\right)^{2}\right.
$$

$-m_{T}^{N}(t)$ is the synchronous averaging;

- $N$ cycle number.

Synchronous variance allows highlighting the periodic irregularities caused, for example, by changes in speed or load. To estimate the synchronous variance, for each cycle the synchronous average is subtracted then squared, and then averaged over all cycles.

\subsection{Spectral correlation}

In this work, the spectral correlation will be used for the characterization of cutting process. The spectral correlation is the double Fourier Transform, from $(t, \tau)$ to $(\alpha, f)$ of the autocorrelation function $R_{2 x}(t, \tau)[18]$ :

$$
S_{x}(\alpha, f)=\lim _{W \rightarrow \infty} \iint_{-\frac{W}{2}}^{\frac{W}{2}} R_{2 x}(t, \tau) \mathrm{e}^{-j 2 \pi \alpha t} \mathrm{e}^{-j 2 \pi f \tau} \mathrm{d} t \mathrm{~d} \tau
$$

The autocorrelation function of the signal depends on two parameters: the time $t$ and delay $\tau$. The frequency obtained by FT (Fourier Transform) versus the variable $\tau$ is the spectral frequency $f$. That obtained by FT according the variable $t$ is called cyclic frequency and denoted $\alpha$.
For a second order cyclostationary signal, the spectral correlation is continue by $f$, (since the autocorrelation function is random by $\tau$ ) and discrete according to the cyclic frequency $\alpha$ since the autocorrelation function is periodic by $t$ (not null for $\alpha=\frac{k}{T}$ with $k \in Z$ ). For a stationary signal the spectral correlation according to the cyclic frequency is zero for $\alpha \neq 0$. So the spectral correlation can reveal the presence of the first and second order cyclostationarity. For the estimation of spectral correlation the averaged cyclic periodogram is used [18].

\section{The machining operation modeling}

\subsection{The mechanism milling operations}

During the milling operation, the tooth of the cutter is subjected to two force components: tangential and radial (Fig. 2). To maintain a constant machining rate, the instantaneous tangential force applied on a single tooth of the cutter is given by Equation (7):

$$
F_{t}=K_{s} a S_{t} \sin \theta+a C_{w} V B, \quad \theta_{\text {entry }} \geq \theta \geq \theta_{\text {exit }}
$$

And the radial force is given by Equation (8):

$$
F_{r}=r_{1} K_{s} a S_{t} \sin \theta+r_{2} a C_{w} V B, \quad \theta_{\text {entry }} \geq \theta \geq \theta_{\text {exit }}
$$

where $K_{s}$ is the specific pressure constant, $a$ is the depth of cut, $S_{t}$ is the feed per tooth, $\theta$ is the instantaneous angle, $C_{w}$ is the force constant of the edge, $r_{1}$ et $r_{2}$ are constant related to the force rates and $V B$ is the average width of flank wear.

For a sharp tool (without wear), the two equations are simplified to:

$$
\begin{gathered}
F_{t}=K_{s} a S_{t} \sin \theta \\
F_{r}=r_{1} K_{s} a S_{t} \sin \theta
\end{gathered}
$$

The model given by Equations (9) and (10) can be modeled by a signal of the form: $a(t) \sin (t)$, which is a cyclostationary signal [19], where $\sin (t)$ presents the periodic component (spindle rotation) and $a(t)$ presents a random white noise with non-null mean which corresponds to the mean depth. This random signal characterizes the variation due to section thickness (the variable $a S_{t}$ ). Thus the signals $\left(F_{r}(\theta)\right.$ and $\left.F_{t}(\theta)\right)$ are cyclostationary if the speed fluctuations of the rotation spindle are small or if the signal is angular sampled [16].

\subsection{Signal vibration model}

For a vibration signal measured by an accelerometer sensor in a given direction, we can give the following model:

$$
x(n)=\sum_{k=-\infty}^{k=\infty} F(k) h_{i}(n-k)
$$

with $F(n)$ the excitation which is the applied cutting force, $h_{i}(n)$ is the impulse response. This impulse response 

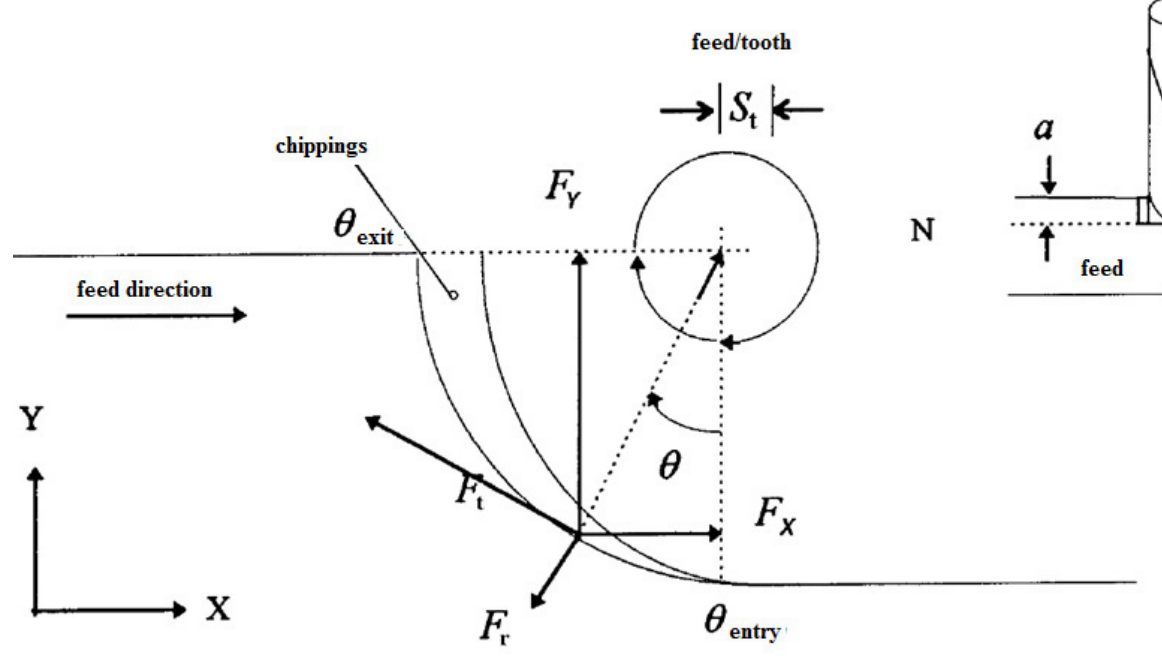

Fig. 2. Schematic representation of the cutting forces in an end milling operation.

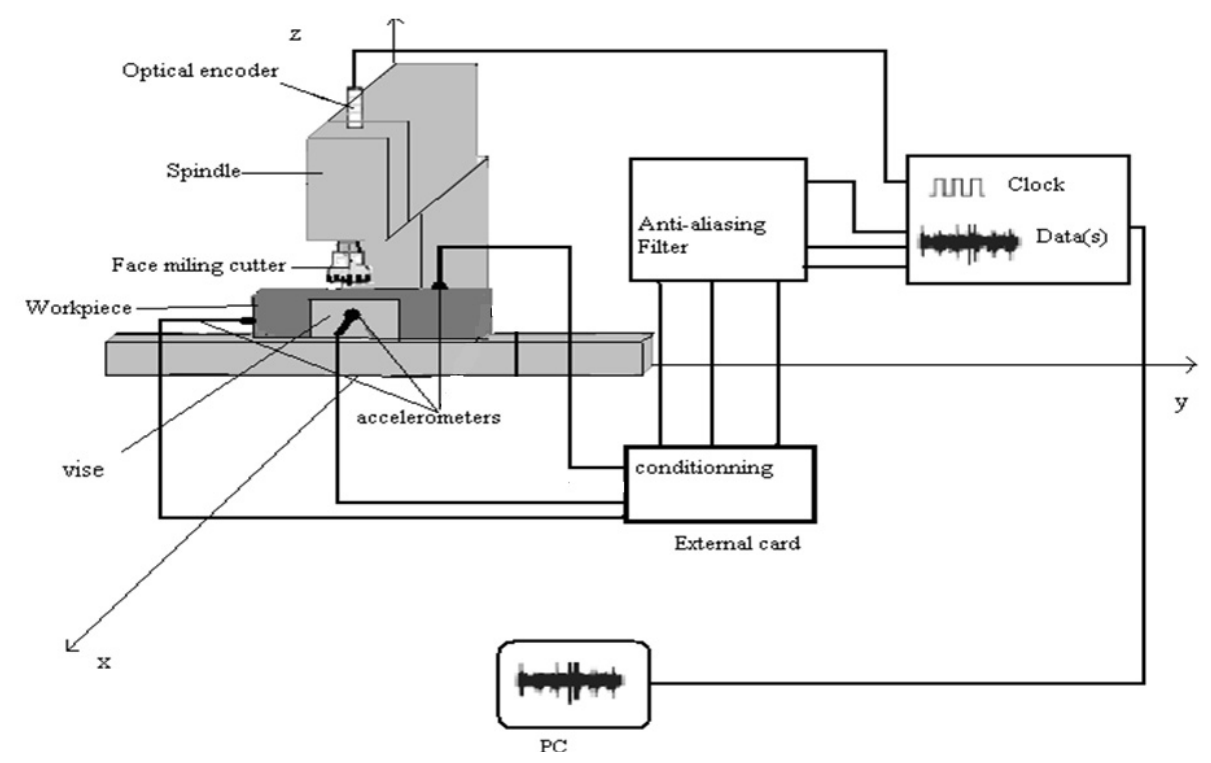

Fig. 3. Schematic presentation of the experimental setup.

depends on the sensor position $i$. From Equation (11) it can be seen that the vibration signal captured from a milling operation is cyclostationary if the signal is angular sampled or if the speed fluctuations are small.

\section{Test rig}

To use the method presented in this paper in the monitoring, a test rig to generate cutting data from a milling operation was prepared. The test rig composed of a milling machine type Cincinnati equipped with a motor (Fig. 4a). Accelerometers (PCB model A601A01, gain $100 \mathrm{mv} / \mathrm{g}$ and weight $80 \mathrm{gm}$ ) were placed, with a magnetic mounting, in three mutually perpendicular directions
( $x$ direction, $y$ work piece, $z$ direction as shown in Fig. 3 ). An optical encoder to enable the angular sampling was installed at the spindle, (Fig. 3). This delivers a position information (squared signal at frequency of 2500 squares per rotation period), which is used as a clock for the data acquisition card. Therefore, signals were sampled at constant angle intervals. For an optical encoder of a resolution equal to 2500 points per revolution, and an average speed of revolution $f r=8.33 \mathrm{~Hz}$, the average sampling frequency will be $f_{s}=f_{r} 2500$. The Nyquist frequency must be adjusted according to the instantaneous speed (the instantaneous sampling frequency depends to the instantaneous speed). Experiments with the milling cutting tool were performed for $40 \mathrm{~s}$ of milling. The face milling cutter had 5 unequally spaced teeth. The cutting 
Table 1. Cutting conditions and angular sampling parameters.

\begin{tabular}{ll}
\hline Material of the specimen & Steel \\
Optical encoder resolution & 2500 point per revolution \\
Number of recorded cycles & 300 \\
Number of samples & 750000 \\
Averaged sampling rate & $20834 \mathrm{kHz}$ \\
Anti-aliasing filter & $8 \mathrm{kHz}$ \\
Cutting condition & Feed speed: $250 \mathrm{~mm}$ \\
& Cutting depth: $0.7 \mathrm{~mm}$ \\
Number of data & Free fault: 3 acquisitions \\
acquisitions & a broken tooth: \\
& 3 acquisitions \\
\hline
\end{tabular}

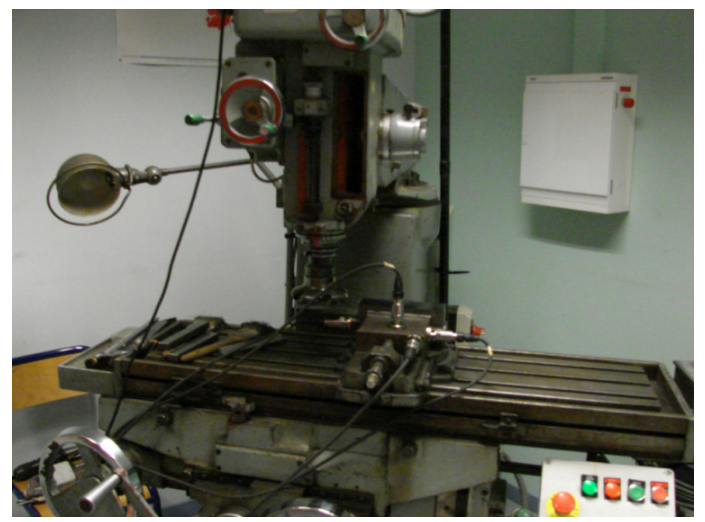

(a)

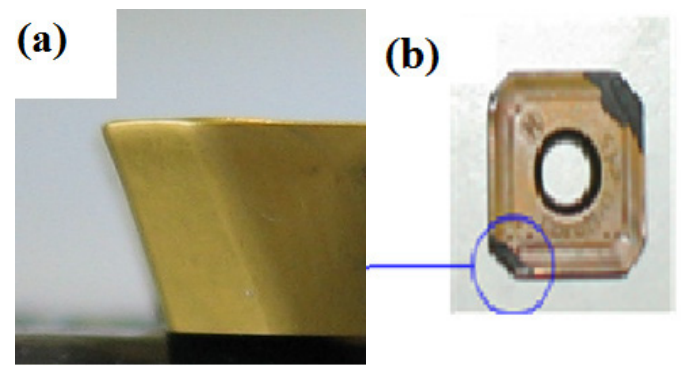

(b)

Fig. 4. (a) the used milling machine. (b) the used tool tips: (a) new, (b) has a broken edge.

parameters and the operating conditions were kept constant during the experiment (see Tab. 1). In the experimental analysis, we have considered two tool cutting states: free fault and with a broken tooth.

\section{Signal analysis}

\subsection{Synchronous statistics}

This paragraph concerns the analysis of the signals that were captured from the test rig. Figure $5 \mathrm{a}$ presents three cycles of the signal from accelerometer $1(\times$ direction), Figure 5b shows three cycles of the signal from accelerometer 2 ( $y$ direction) and Figure $5 c$ three cycles of the signal from accelerometer 3 ( $z$ direction) for free fault case (test 1). All the figures show the repetition of the five peaks which correspond to five teeth on the cutter. The amplitude of the peaks is not equal due to the teeth being unequally spaced. The choice of cutter with unequally spaced teeth is often made by the manufacturers in order to limit the vibration level. The repetition noticed in Figure 5 shows the cyclostationary nature of the milling vibration signal taken under angular sampling. This observed characteristics will motivate us to check the cyclostationary at different order.

To study the first order cyclostationarity, it is necessary to estimate the periodic component. This periodic component is estimated by the synchronous averaging. The use of synchronous averaging allows us to overcome the problems caused by speed variations. The synchronous statistics give the information versus the position. Figure 6 shows the synchronous averaging estimated on 100 cycles in the free fault case and a broken tooth case. The position of the broken tooth is on the second position after top zero (angular position $n=844$ ), one can notice the peak disappearance in this angular position. For the accelerometer 2 and 3 we see the same manifestation.

Figures $7 \mathrm{a}-7 \mathrm{c}$ present the estimated synchronous variance of the milling operation in the cases of faulty case and free fault case for the three accelerometers (1-23 ). In these figures one can observe the presence of the 5 peaks which interpret the variability between cycles. These peaks are more obvious in synchronous variance than in the synchronous averaged. In the faulty case we have loss of the peak which corresponds to the broken tool tip and the amplitude of the three peaks, which follow the broken tooth, become higher. These amplitudes increase is owing to the fact that the next tool tips must remove more the metal. We notice that the tooth is completely broken and the edge of the broken tooth does not touch the workpiece, that why in the rank of work of the broken tooth the synchronous variance is zero. We also find that the shape and amplitude of peaks depend on the sensors location and that all these sensors flagrantly reveal the presence of the broken tooth.

In conclusion, the synchronous variance shows that there is variability from one cycle to the other and this variability is periodic with respect the rotation period of the spindle. In other word, the signal is second order cyclostationary. Also the synchronous variance amplitude allows the detection of the fault and localizes its emplacement.

\subsection{Angular sampling effect}

Figure 8a presents the synchronous variance of a signal of a milling operation that is temporally sampled (by the dynamic acquisition system' OROS'). As shown in this figure the synchronous variance of the temporal signal is constant (equal to $22\left[\mathrm{~m} / \mathrm{s}^{-2}\right]^{2}$ ), which corresponds to the case of a stationary signal. Figure $8 \mathrm{~b}$ shows the comparison, between the temporal signal after angular 

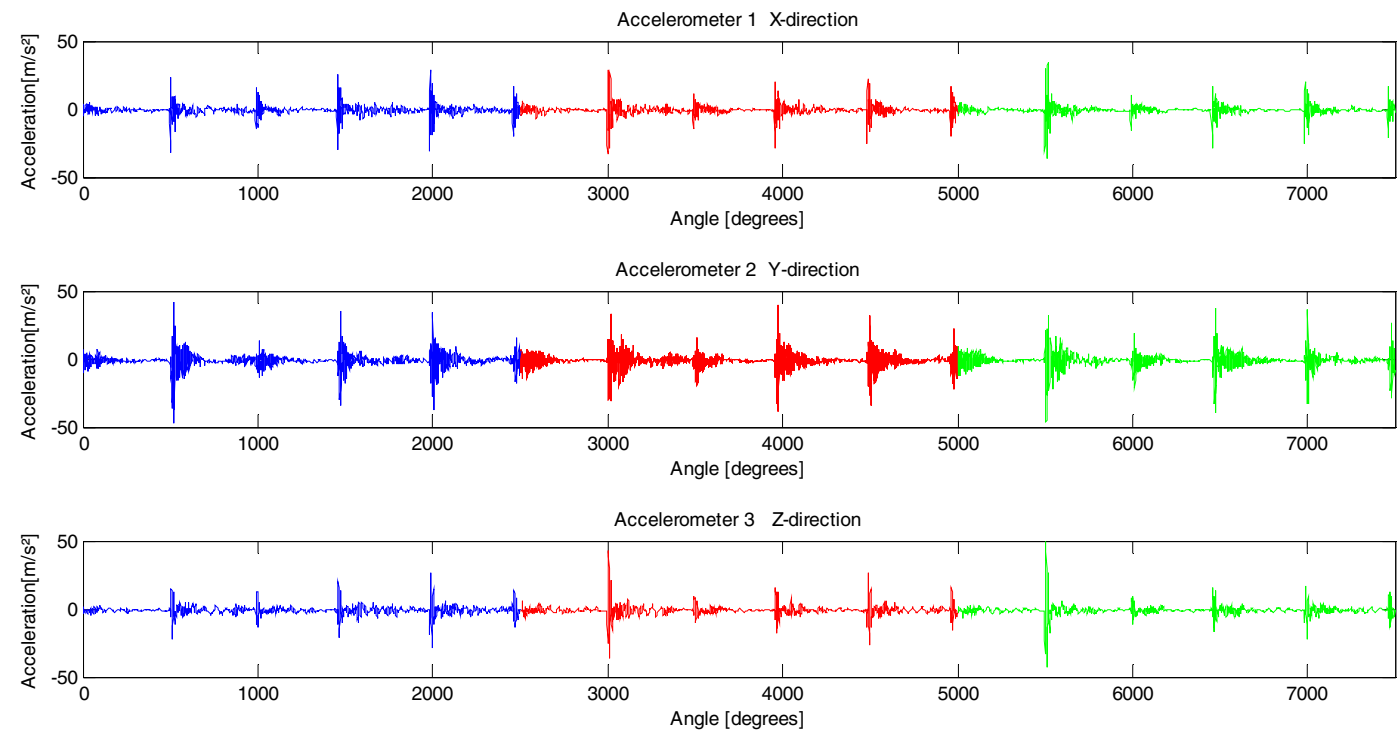

Fig. 5. Typical milling signals.

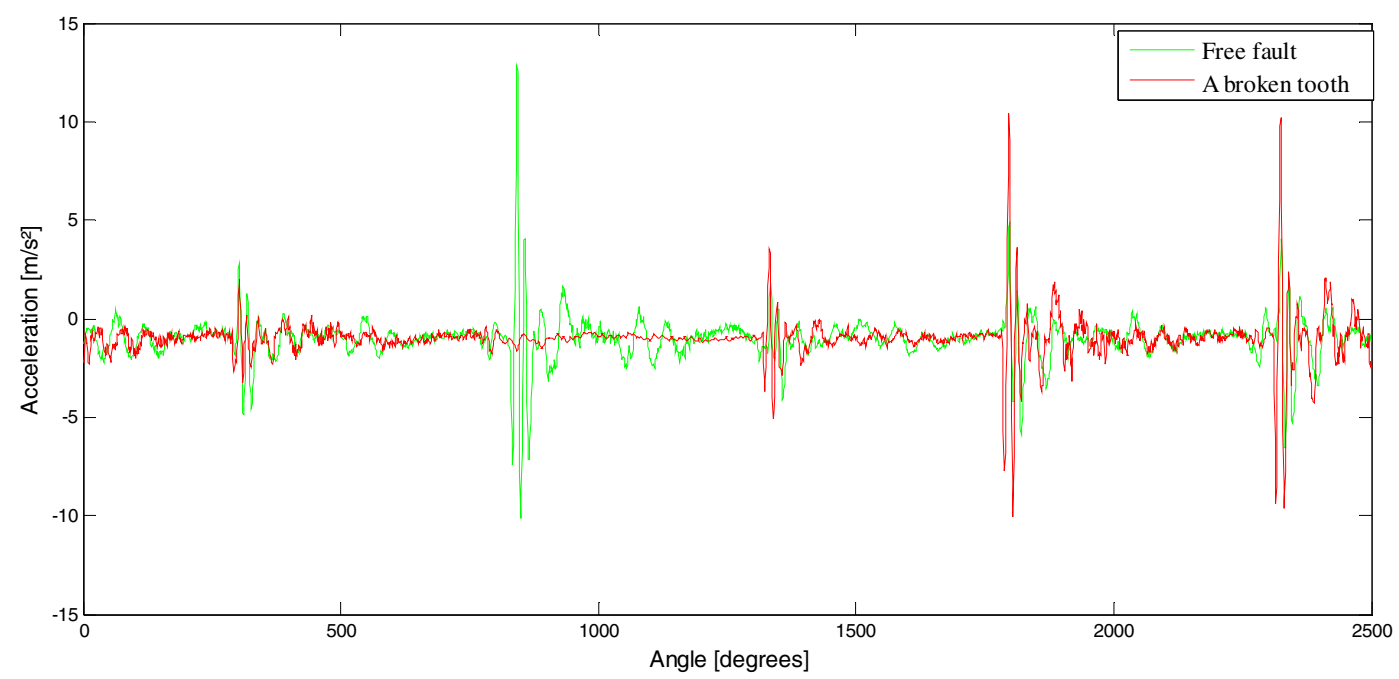

Fig. 6. Synchronous averaged [100 averages] of signal coming from the test No. 1 accelerometer 3 for free fault and a broken tooth cases.

resampled by the algorithm presented in [20] and a signal that is direct angular sampled (as in the test rig part). In the case of angular resampled signal, we find the peaks which correspond to each tooth. Also these peaks are of the same amplitudes and emplacements than the direct angular sampled signal. Thus, it can be seen from these two figures that the angular resampling presents a potential alternative that can be used to save the cyclostationary characteristics of a signal which the supplementary information, given by the cyclostationary property, is destructed by the speed fluctuations.

\subsection{The spectral correlation}

The above results validate the cyclostationarity aspect of the vibration signals, of a milling cutting operation, that are angular sampled. This validation is done with the synchronous statistics. In this section, the spectral domain is analyzed in the cyclostationary context. The key tool for this is the spectral correlation (or spectral correlation density). The spectral correlation estimated from the cumulants (after extraction of the synchronous averaged) shows the purely second order cyclostationary. The extraction of the first order cyclostationary before the spectral correlation estimation is equivalent to the mean value extraction, in the case of signal stationary, for the power spectral density. That eliminates the first order effect which may mask the information given by the second order.

The estimation method of the spectral correlation is the averaged cyclic periodogram [18]. The calculation parameters are: the signal size is $2500 \times 100$ samples. This signal is then decimated by factor 2 and the sampling frequency is $10417 \mathrm{~Hz}$. The window size is 5000 points, the 


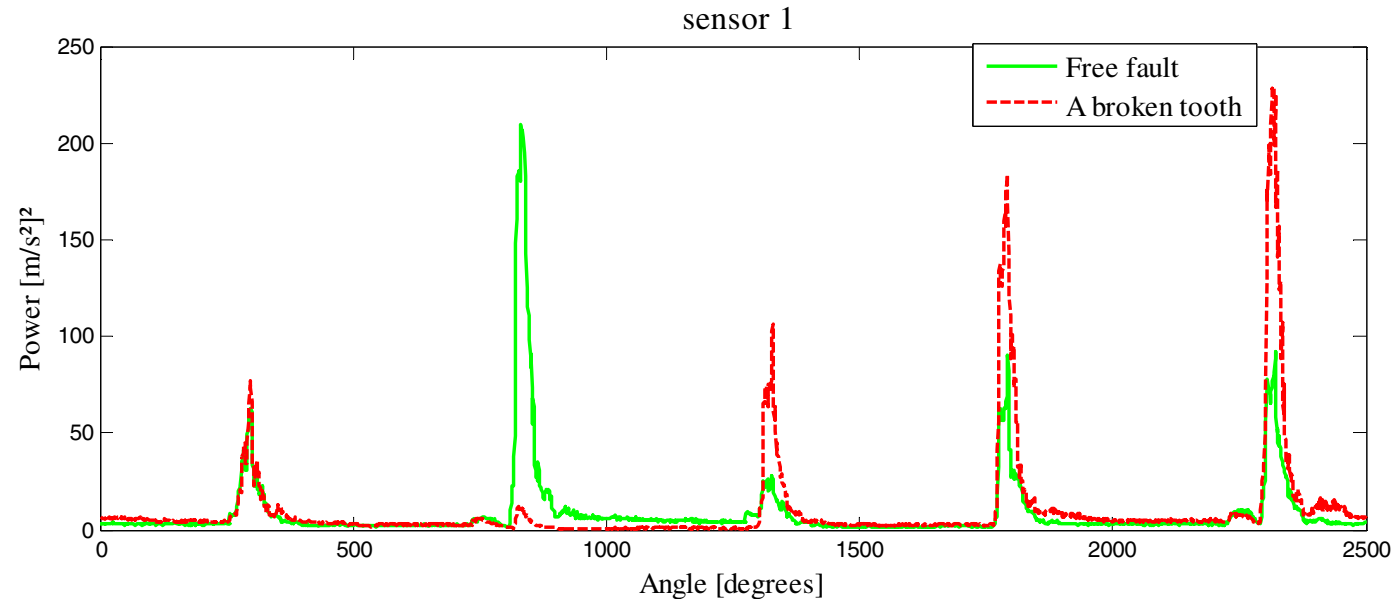

(a)

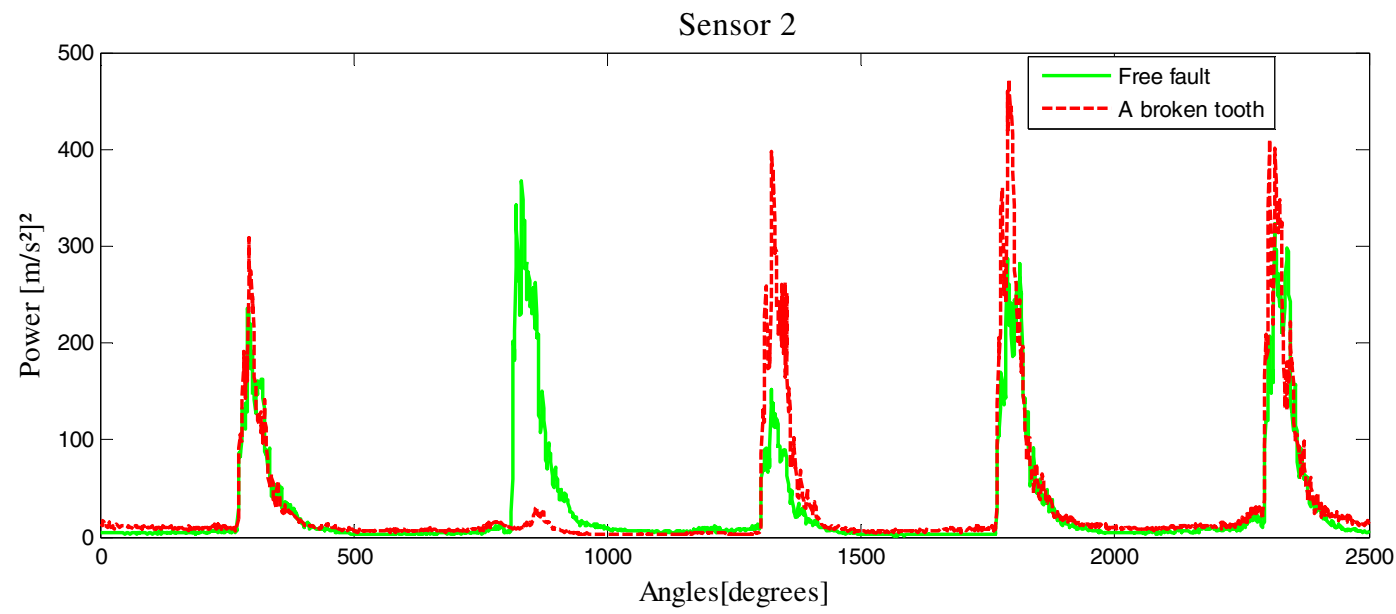

(b)

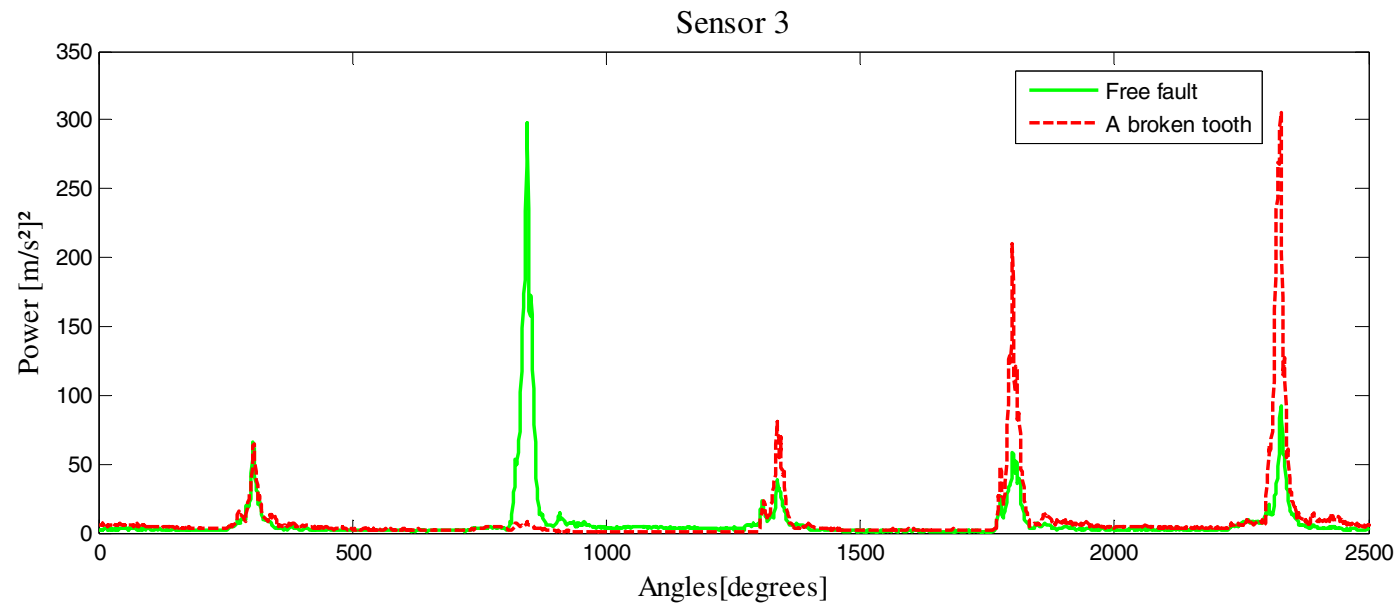

(c)

Fig. 7. Synchronous variance (estimated on 300 cyles) of signal coming from the test No. 2 for free fault and a broken tooth cases, (a) sensor 1, (b) sensor 2 and (c) sensor 3.

overlap is $75 \%$ and the window type is Hanning. Figure 9 presents the spectral correlation of accelerometer 1 in the case of free fault. In this figure we can see the spectral lines that are repeating by the frequency cyclic. It is noted that the 5th spectral line and its harmonics are more energetic than the other spectral lines. This cyclic frequency corresponds to the frequency tooth passing (milling cutter with 5 teeth). Figure 10 presents the spectral correlation in the case of a broken tooth. We can find that the cyclical modulation spectrum, along the cyclic frequency axis, changes in the presence of the defect. We have the spectral line amplitudes increase significantly above the rotation frequency and its harmonics. This increase is greater in the frequency band [2000-4000 Hz]. This result is consistent with the milling machine kinematics as the fault occurs at the rotation frequency of the spindle. Also, 


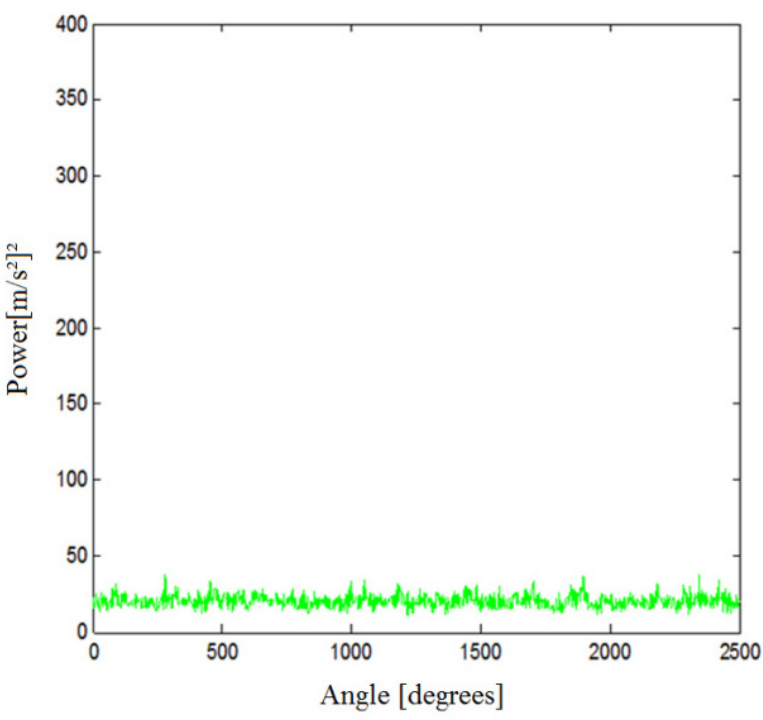

(a)

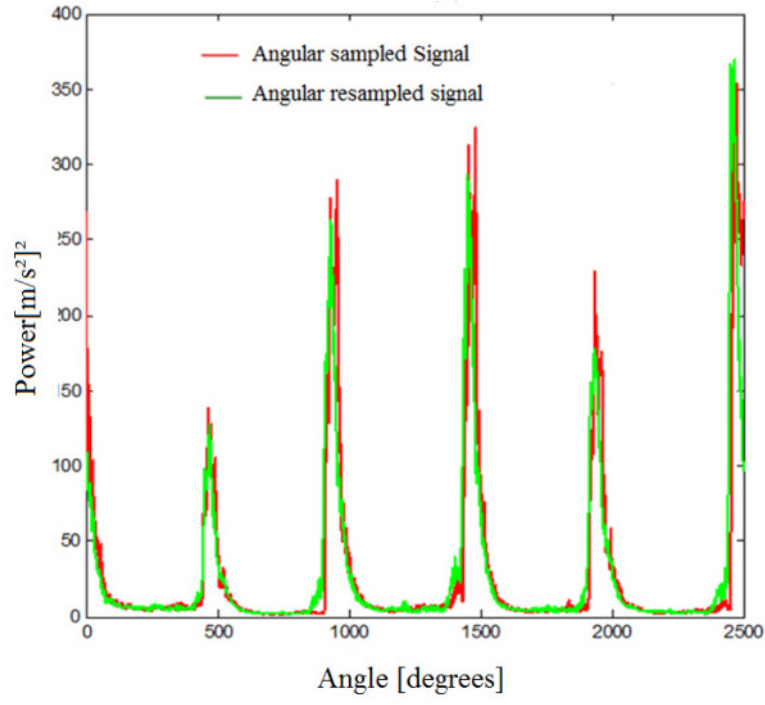

(b)

Fig. 8. (a) synchronous variance of the temporally sampled signal. (b) Synchronous variance of the angular resampled signal [20] (green), synchronous variance of the angular sampled signal (red).

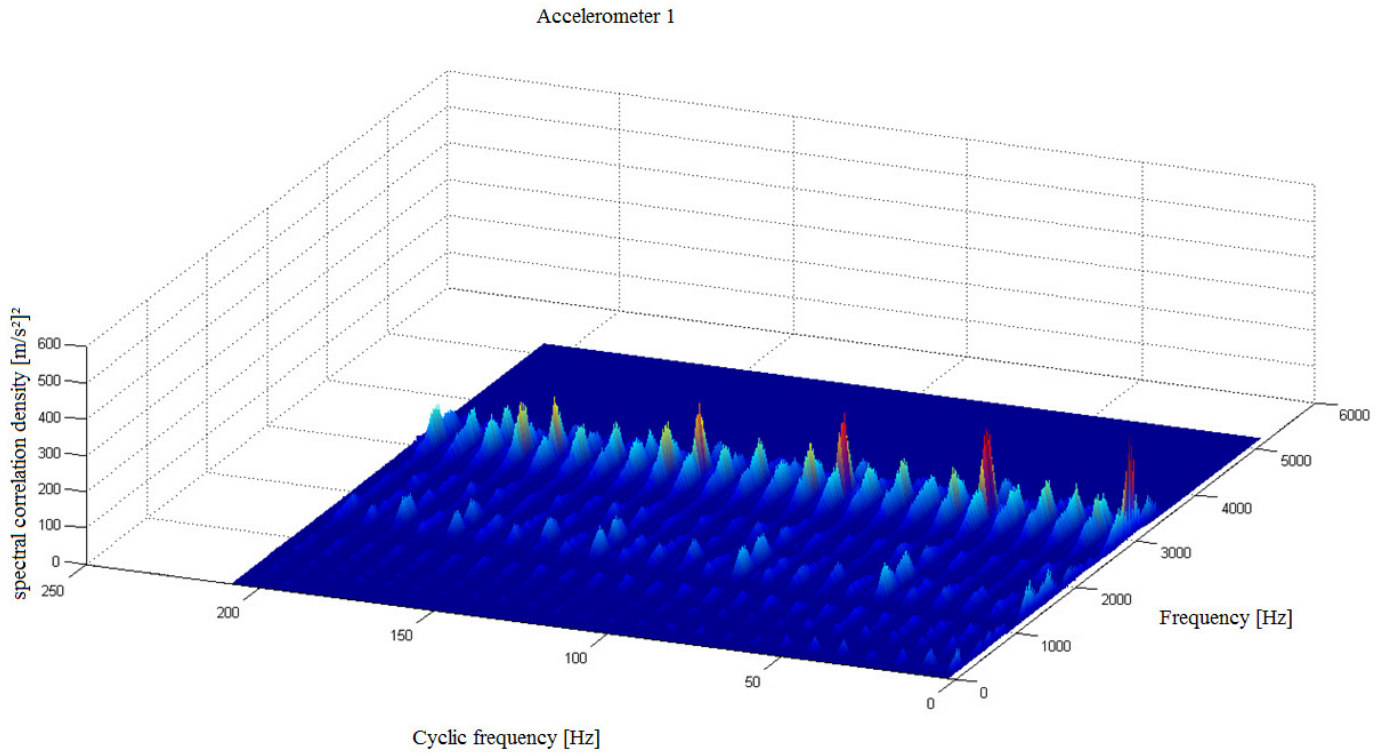

Fig. 9. Spectral correlation density of vibration signal, coming from the accelerometer 1 and test 1 , in the case of free fault.

according to the cyclic frequency, we can see that three most energetic spectral lines follow two spectral lines that have less energy. This is explained by the fact that the three teeth after the broken tooth work more. These results corroborate the results obtained in the previous paragraph. Nevertheless, they provide us additional information about the fact that the signal is polycyclostationary. Indeed, we have two cyclic frequencies: a cyclic frequency associated with the spindle rotation, and another associated with the tooth passage. The spectral correlation can be used to characterize the signal in a first approach. For the diagnosis it is not practical and it is preferable to use the envelope analysis which is easier to estimate and interpret. The link between the spectral correlation and the envelope analysis was established by Antoni and RB Randall [21], they showed that the envelope analysis is the same as to project the spectral correlation by the cyclic frequency axis.

In order to exploit the second order cyclostationarity for the cutting tool diagnosis, we project the spectral correlation by the cyclic frequency axis. The projection is useful to "condense" the information contained in the spectral correlation. The spectral correlation is taken only in a frequency band $[f 1, f 2]$ where the spectral lines increase with the defect. Figures $11 \mathrm{a}-11 \mathrm{c}$ present the projection of the spectral correlation, for three accelerometers, 


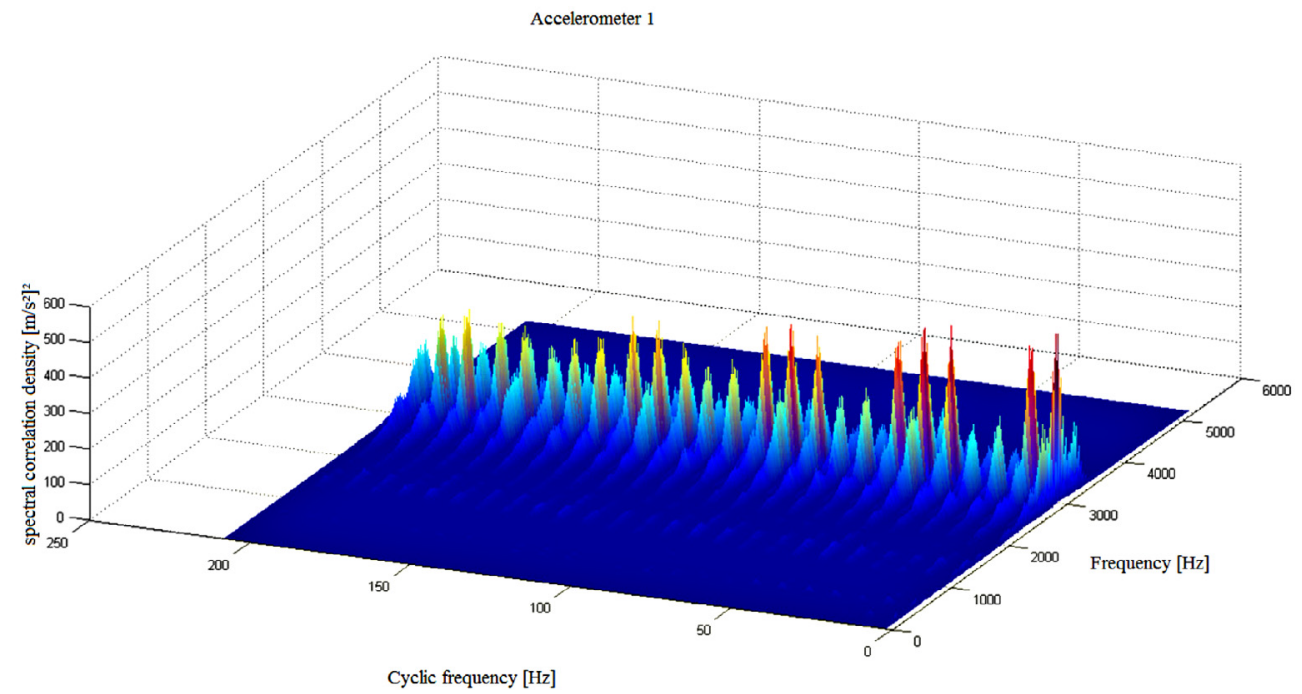

Fig. 10. Spectral correlation density of vibration signal, coming from accelerometer 1 test 1 , in the case of one broken tooth.
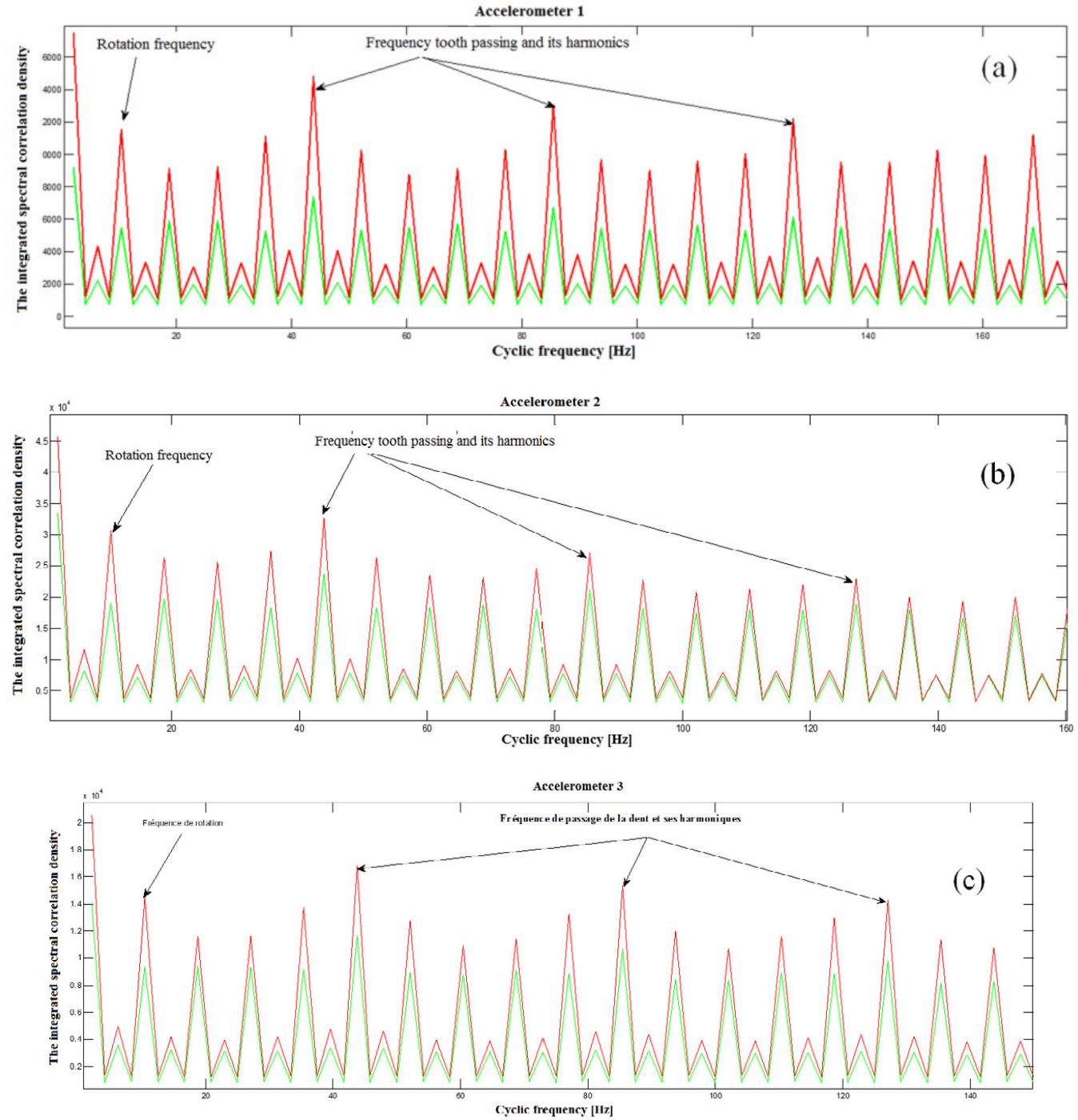

Fig. 11. The integrated spectral correlation density: (a) accelerometer 1 [2000-4000 Hz], (b) accelerometer 2 [2000-3000 Hz], (c) accelerometer $3[800-2100 \mathrm{~Hz}]$. 
by the cyclic frequencies in the following frequency bands ([2000-4000 Hz] for the sensor 1, [2000-3000 Hz] for the sensor 2 and $[800-2100 \mathrm{~Hz}]$ for the sensor 3 ), in the cases of free fault and a broken tooth. We find that the modulation peaks has changed in the presence of the defect. Also the amplitudes of rotational frequencies have increased in the case of the faulty case. This increase is significant and revealing the defect presence. We see that it varies between different sensors.

To better exploit the information contained in the integrated spectral correlation, we propose an indicator $I_{1}$ that is given by the following equation:

$$
I_{1}=\int_{\alpha_{i}=0}^{\alpha_{i}=N f r} \int_{f 1}^{f 2}\left|S_{x}\left(\alpha_{i}, f\right)\right| \mathrm{d} f \mathrm{~d} \alpha
$$

with $f 1$ and $f 2$ the frequency band in which the spectral correlation is integrated, $N$ the number of cyclic frequencies taken into account and $f_{r}$ the cyclic frequency. This indicator consists in integrating the projected spectral correlation on the cyclic frequency. Figure 12 shows this indicator for $N=20$ for the various tests made during the experiment.

It can be seen from this figure that the values of $I_{1}$ for different tests in the free fault case are very similar; for example the sensor 1 , the value does not exceed $I_{1}=1 \mathrm{e}+$ 006 for all tests, whereas in the case of a broken teeth is more than $I_{1}=1.26 \mathrm{e}+006$. Thereof we can set a threshold which allows the classification of cutting tool state. The chosen threshold can be for example the middle, between the average values of $I_{1}$ for all tests in the free fault case and with defect case. For sensor 1 , this threshold value is $I_{1}=1.17 \mathrm{e}+006$.

\section{Conclusion}

The work purpose is the diagnosis of the milling machine in order to control the cutting tools breakage and therefore the machining process. There are many approaches to improve the performance and availability of the production process. The proposed approach is based on the vibration analysis of the milling machine. In the literature, most research works on the detection of the cutting tool failure assume that the vibration signals are stationary and therefore they use classical tools like power spectral density or global statistical indicators such as the kurtosis, RMS, etc. Our approach is based on physical considerations of the machine operation which allowed us to check in a first time that signals captured from a milling machine are cyclostationary. In a second time we explicitly included this property in the proposed signal processing tools used to best detect a tool tip failure.

In other word, the approach consists of performing an angular sampling to enhance the cyclostationary nature of signals. Subsequently, the estimation of synchronous statistical moments allowed us to validate the cyclostationary property of signals. These statistics also allowed us, through the synchronous variance, the detection of the tool breakage presence and its location.

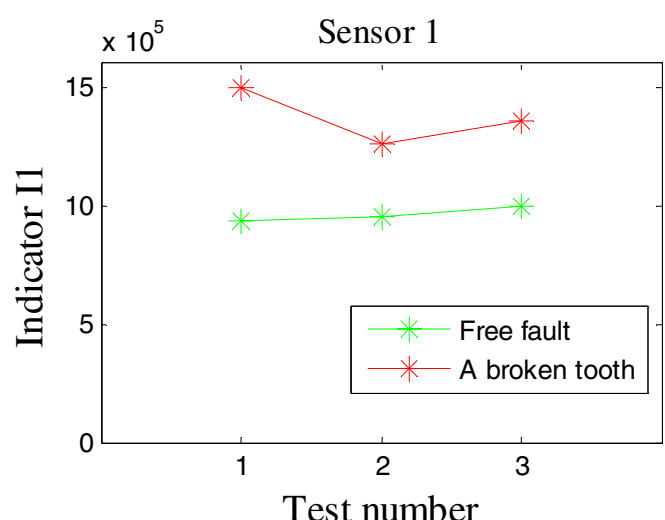

(a)

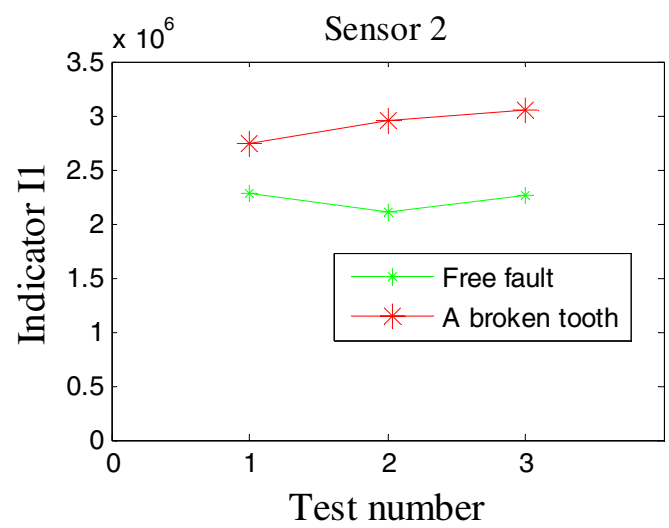

(b)

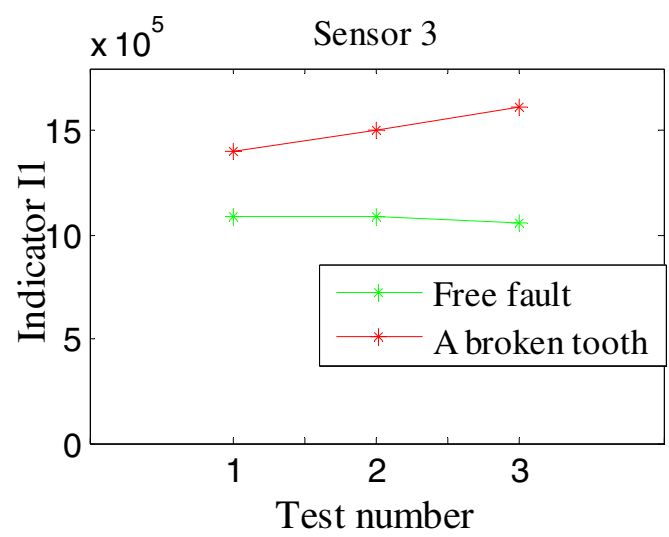

(c)

Fig. 12. Indicator $I_{1}$ (a) accelerometer 1, computed on frequency band [2000-4000 Hz], (b) accelerometer 2, computed on frequency band [2000-3000 Hz], (b) accelerometer 3, computed on frequency band [800-2100 Hz].

The spectral correlation allows the detection of the tool tips breakage presence and also highlights the different cyclic frequencies which are specific to the machine kinematics (the rotation frequency and the frequency of tooth passage). Therefore the signals are polycyclostationary. In the free fault case, we can see versus the cyclic frequency that the amplitude of the spectral line 
at frequency tooth passing is greater than the amplitude of the spectral line at the rotation frequency. This result is explained by the fact that the milling cutter has unequally spaced teeth. This has the effect of generating a random phase modulation which is superimposed on the inherent cyclostationarity to the spindle rotation. This phase modulation is modified in the presence of the tool breakage. Also from the spectral correlation we proposed an indicator that can be used to detect the presence or not of the tool tip breakage.

\section{References}

[1] G. Byrne, D. Dornfeld, I. Inasaki, G. Ketteler, W. König, R. Teti, Tool Condition Monitoring (TCM) - The Status of Research and Industrial Application, CIRP Annals: Manuf. Technol. 44 (1995) 541-567

[2] S.H. Yeo, L.p. Khoo, S.S. Neo, Tool condition monitoring using reflectance of chip surface and neural network, J. Intelligent Manuf. 11 (2000) 507-514

[3] S. Kurada, C. Bradeley, A review of machine vision sensors for tool condition monitoring, Comput. Ind. 34 (1997) 55-72

[4] A.G. Rehorn, J. Jiang, P.E. Orban, State-of-the-art methods and results in tool condition monitoring: a review, Int. J. Adv. Manuf. Technol. 26 (2005) 693-710

[5] R.L. Kegg, On-line machine and process diagnostics, CIRP Annals: Manuf. Technol. 33 (1984) 469-473

[6] S.N. Huang, K.K. Tan, Y.S. Wong, C.W. de Silva, H.L. Goh, W.W. Tan, Tool wear detection and fault diagnosis based on cutting force monitoring, Int. J. Mach. Tools Manuf. 47 (2007) 444-451

[7] E. Kuljanic, M. SortinoTWEM, a method based on cutting forces-monitoring tool wear in face milling, Int. J. Mach. Tools Manuf. 45 (2005) 29-34

[8] F.J. Alonso, D.R. Salgado, Analysis of the structure of vibration signals for tool wear detection, Mech. Syst. Signal Process. 22 (2008) 735-748

[9] S. Orhan, A. Osman, N. Camuscu, E. Aslan, Tool wear evaluation by vibration analysis during milling of AISI D3 cold work tool steel with 35 HRC hardness, NDTE\&E Int. 40 (2007) 11-126
[10] D.E. Dimla, Sensor signals for tool-wear monitoring in metal cutting operations-a review of methods, Int. J. Mach. Tools Manuf. 40 (2000) 1073-1098

[11] J. Lin, Inverse estimation of the tool-work interface temperature in end milling, Int. J. Mach. Tools Manuf. 35 (1995) 751-760

[12] Srinivasa Pai P., Ramakrishna Rao P.K., Acoustic emission analysis for tool wear monitoring in face milling, Int. J. Prod. Res. 40 (2002) 1081-1093

[13] W.A. Gardner, Stationarizable Random Processes, IEEE Trans. Inf. Theory 24 (1978) 8-22

[14] W.A. Gardner, Statistical Spectral analysis: a non probabilistic theory, Prentice, Hall Inc, 1988

[15] C. Capdessus, M. Sidahmed, J.L. Lacoume, Cyclostationary processes, application in gear faults early diagnosis, Mech. Syst. Signal Process. 14 (2000) $371-38$

[16] J. Antoni, F. Bonnardot, A. Raad, M. Elbadaoui, Cyclostationnary modelling of rotating machine vibration signals, Mech. Syst. Signal Process. 18 (2004) 1285-1314

[17] J. Antoni, Apports de l'échantillonnage angulaire et de la cyclostationnarité au diagnostic par analyse vibratoire des moteurs thermiques, Thèse de l'Institut national polytechnique de Grenoble, 2000

[18] J. Antoni, Cyclic spectral analysis in practice, Mech. Syst. Signal Process. 21 (2007) 597-630

[19] M.A. Elbestawi, T.A. Papazariou, R.X. Du, In process monitoring of tool wear in milling using cutting force signature, Int. J. Mach. Tools Manuf. 31 (1991) 55-73

[20] F. Bonnardot, Comparaison entre les analyses angulaire et temporelle des signaux vibratoires de machines tournantes. Etude du concept de cyclostationnarité floue, Thèse de l'Institut national polytechnique de Grenoble, 2004

[21] R.B. Randall, J. Antoni, The relationship between spectral correlation and envelope analysis in the diagnostics of bearing faults and other cyclostationary machine signals, Mech. Syst. Signal Process. 15 (2001) 945-962 